\title{
Investigation of nonlinear effects in Doppler reflectometry using full-wave synthetic diagnostics
}

O. L. Krutkin ${ }^{1,2}$, A.B. Altukhov ${ }^{1}$, A.D. Gurchenko ${ }^{1}$, E.Z. Gusakov ${ }^{1}$, S. Heuraux ${ }^{2}$, M.A. Irzak $^{1}$, L.A. Esipov ${ }^{1}$, T.P. Kiviniemi ${ }^{3}$, C. Lechte ${ }^{4}$, S. Leerink ${ }^{3}$, P. Niskala ${ }^{3}$, G. Zadvitskiy ${ }^{2}$

${ }^{1}$ Ioffe Institute, St Petersburg, Russia

${ }^{2}$ Institut Jean Lamour UMR 7198 CNRS, Univ. de Lorraine, BP 50840, 54011 Nancy, France

${ }^{3}$ Aalto University, Espoo, Finland

${ }^{4}$ Institute of Interfacial Process Eng. and Plasma Technology, 70569 Stuttgart, Germany

\begin{abstract}
In this work, Doppler reflectometry (DR) and radial correlation DR (RCDR) nonlinear scattering effects were studied using full-wave modeling and a set of representative FT-2 tokamak turbulences as inputs. Narrowing of the RDCR correlation function and widening of the DR poloidal wavenumber spectrum are demonstrated. An effect on the dependence of the DR signal frequency shift on the probing wavenumber is found, namely, this dependence "linearizing" in the nonlinear scattering regime. Nonlinear effects are shown to be weaker for O-mode probing than for X-mode probing, while a faster transition to nonlinear regime is demonstrated for RCDR compared to DR in both probing scenarios.
\end{abstract}

\section{Introduction}

Turbulent transport plays a major role in plasma confinement, which makes understanding and control of plasma turbulence one of the main goals of fusion research. The tools for the turbulence characterization include Doppler reflectometry (DR) and radial correlation Doppler reflectometry (RCDR) [1], the two diagnostics based on probing the plasma with a microwave beam at an oblique incidence with respect to magnetic surfaces and measuring the characteristics of the scattering signal to extract information on the turbulence that induced the scattering. RCDR in particular utilizes simultaneous probing with two microwaves at different frequencies incident obliquely onto the magnetic surface in the presence of the cutoff. As it was shown in [2,3], at a large enough incidence angle by performing correlation analysis of backscattering signals, information about turbulence properties, such as the radial correlation length can be extracted, while the conventional DR provides information on frequency and poloidal wavenumber spectra of the turbulence, as well as on the fluctuation rotation velocity in poloidal direction.

However, for both the RCDR and the conventional DR, the analytical theory predicts direct relation of measured quantities to the turbulence characteristics only in the linear regime of scattering [3], corresponding to low turbulence amplitudes. Some analytical results for the nonlinear regime, such as criteria for the onset of the nonlinear behavior $[4,5]$ and for the transition to fully nonlinear regime corresponding to the probing wave extinction due to its strong phase modulation by the turbulence and strong wave-front distortion were derived. A 
saturation of the scattering signal power growth with the turbulence level was obtained in this regime for the radial correlation reflectometry [6,7] and for DR [8]. Nevertheless, the interpretation of experimental DR and RCDR measurements is challenging in the nonlinear regime and in the transition to it. Moreover, even in the linear regime, due to a substantial contribution of the small-angle scattering off long-scale fluctuations in the reflected signal, the turbulence radial correlation length could be overestimated [3,9]. Methods were, however, suggested to amend this issue and to reconstruct the turbulence radial wavenumber spectrum based on RCDR data [10, 11].

Overall, the mentioned difficulties of the reflectometry data interpretation make full-wave numerical modeling one of the main tools of analysis of RCDR and DR. Numerical studies of RCDR and DR were performed [12] and recently, synthetic diagnostics which allow comparing the gyrokinetic modeling results directly to the experimental measurements were developed [13-[16]. One such diagnostic was developed for FT-2 tokamak [13] and demonstrated a good agreement with the experimental data. As this synthetic diagnostic was created, extensive full-wave computations using two-dimensional arrays of density values computed by a Gyrokinetic code (GK) were carried out. This work highlights the results of these computations focusing on the effects of the nonlinear scattering on DR and RCDR measurements.

\section{The computation approach}

Parameters of the FT-2 ohmic discharge used for the gyrokinetic modeling are as follows: $B_{0}=1.7 \mathrm{~T}, I_{p}=19 \mathrm{kA}, n_{e}^{m a x}=4.2 \cdot 10^{19} \mathrm{~m}^{-3}$, while the major and minor radii of the device are $0.55 \mathrm{~m}$ and $0.08 \mathrm{~m}$ respectively. More detailed information on the discharge as well as on experimental profiles can be found in [13],[15]-[17], as well as schematics and illustrations of the DR configuration used. Same as in experimental cases [13],[15] [16] the X-mode probing was performed by a horizontal antenna located at the high-field side (HFS) of the tokamak, which could be shifted vertically from the equatorial plane by up to $2 \mathrm{~cm}$, making the probing oblique and allowing a scan over poloidal wavenumbers of the probing wave. The central probing frequency used was $f_{0}=70 \mathrm{GHz}$, which corresponds to cutoff located at $r=5.5 \mathrm{~cm}$. Such a setup enabled the probing of the turbulence with poloidal wavenumbers of up to $k_{\theta}=12 \mathrm{~cm}^{-1}$. For O-mode probing, a vertically aligned antenna shifted from the chamber axis along the major radius by $5 \mathrm{~cm}$ towards the HFS was used (once again reproducing the experimental configuration in [17]). The probing was performed at the central frequency $f_{0}=30 \mathrm{GHz}$ with the cutoff also being located in the vicinity of $r=5 \mathrm{~cm}$. The turbulence 
wavenumber probed in this case takes the value $k_{\theta}=9 \mathrm{~cm}^{-1}$. Beam diameter of $2 \mathrm{~cm}$ was utilized for both polarizations.

This discharge was modeled by ELMFIRE GK code [18] and the resulting density profile was used for the synthetic RCDR diagnostic. ELMFIRE is a global particle-in-cell code that computes the full distribution function of gyro-kinetic ions and drift-kinetic electrons. The particles are initialized according to a prescribed temperature and density profiles. The profiles are allowed to develop self-consistently in time, as turbulence develops and heat sources and sinks, namely Ohmic heating and radiation losses, are applied. The Ohmic heating is induced by a (spatially homogeneous loop voltage, while electrons are cooled using a Monte Carlo model according to an impurity radiation loss distribution measured by the bolometry diagnostics. The initial oxygen $\mathrm{O}^{6+}$ impurity density is chosen in order to provide the experimental value of $Z_{\text {eff }}=2.2$, as determined using ASTRA modeling and experimental loop voltage measurements, with equal temperatures for the impurities and main ions. Neumann and Dirichlet boundary conditions are used at the boundary of the simulation domain for the electrostatic potential. Particles passing the boundary are returned to the simulation domain as an electron-ion pair according to a probability distribution proportional to the measured radial profile of neutral hydrogen density and at the wall temperature. The specifics of the modeling approach, such as implementation of sinks, sources and collisions can be found in [20]. The temporal dependence available has the duration of $150 \mu$ s with the step of $0,15 \mu \mathrm{s}$ (after being averaged over ELMFIRE intrinsic time step).

The main computation used for further full-wave modelling was performed on a cylindrical grid with 100x8 radial $(r)$ and toroidal $(\phi)$ cells respectively, while number of poloidal $(\theta)$ cells, which adjusts the grid size to local Larmor radius, scaled with $r$ up to 950 at $r=7 \mathrm{~cm}$. This allowed for the cell size of $0.07 \mathrm{~cm}$ (in radial and poloidal direction) in the probing area. Such poloidal resolution allows for the value $k_{\theta \max }=47 \mathrm{~cm}^{-1}$ to be resolved by the code at the studied minor radius, which in turn allows for the value of $k_{\theta} \varrho_{s}=2.6$, where $\rho_{s}=\sqrt{T_{e} / 2 T_{i}} \rho_{i}$. and $\varrho_{i}$ is ion Larmor radius. These parameters mean that ELMFIRE can be used to model trapped electron mode (TEM) instability, which was shown to be typical for FT-2 tokamak [21]. On the other hand, small-scale electron temperature gradient (ETG) instability cannot be correctly resolved by ELMFIRE, which is not a problem as the wavenumbers probed by DR are limited by $k_{\theta} \varrho_{s}=0.7$, placing ETG mode outside of the accessible wavenumber range. Further details on the FT-2 tokamak turbulence and its modeling with ELMFIRE can be found in previous papers [13],[15],[16]. 
Aside from an output of the computation based on experimental profiles, we also used the output of another ELMFIRE run with a temperature profile, which did not correspond to the experimental profile as input. This non-experimental profile, while possessing the same peak value, had a flatter shape resulting in an overestimation of the electron temperature in the probing wave cut-off region (220 eV instead of actual experimental value of $100 \mathrm{eV}$ ). The grid in the poloidal direction had 150 points at any minor radius whereas the turbulence obtained in this case possesses a steeper poloidal wavenumber spectrum. While less relevant for the FT-2 tokamak comparison to experiment, this case was expected to provide clearer indication of nonlinear effects influencing the DR frequency spectra compared to the experimental one while still using a realistic turbulence. This is connected to the fact that the linear signal is proportional to the components of spectrum with higher $k_{\theta}$ while nonlinear one is caused by multiple scattering off the lower $k_{\theta}$ turbulence $[4,5]$.

The realistic density profile, produced with ELMFIRE code for two different profiles of electron temperature was then separated into the background density and the density perturbations by averaging over the window of $30 \mu$ s. Those perturbations were multiplied by a constant factor $a$ to perform the scan over the turbulence amplitude and observe previously mentioned nonlinear effects coming into play.

Obtained background density along with density fluctuations were used as the input for the full-wave code IPF-FD3D [14]. The code was used in its 2D configuration to compute the scattered signal received by the antenna. The configuration used was copied from the experiment. The DR signal calculation was performed separately for each time step provided by ELMFIRE (assuming the turbulence to be frozen), which seems appropriate considering the slow scale in time of the drift-wave $(\mu \mathrm{s}<)$ compared to wave propagation timescale $(\sim \mathrm{ns})$. As a result of the computation for every $0.15 \mu$ s time step obtained from ELMFIRE, the temporal dependence of the complex backscattering signal was obtained. From this dependence a cross-correlation function $(\mathrm{CCF})$ of scattered signals corresponding to the probing at different frequencies was computed. DR spectra were also computed by performing Fourier transform in the windows of $12 \mu$ s averaged over the full $150 \mu$ s of the computation and each toroidal cross-section. The synthetic CCF and DR spectra as well as the total signal power were used for the analysis.

\section{X-mode DR modeling results and analysis}

Due to the strong dependence of its refractive index on the plasma density, X-mode is more susceptible to nonlinearity, which made it the prime object of this study. We will start by 
presenting the non-experimental temperature profile case, extensive computations for which were performed in the $\mathrm{X}$-mode case, as it exhibits the nonlinear effects much clearer.

An example of the DR frequency spectra for a number of different turbulence amplitudes (with $\Delta f$ corresponding to the frequency shift from $f_{0}$ ) is plotted in the Fig. 1, while Fig. 2 demonstrates the dependence of the total scattered power received by the antenna. Fig. 2 is plotted against the dimensionless factor $a$ by which the amplitude of the turbulence produced by ELMFIRE code is multiplied. That means that $a=1$ corresponds to the original ELMFIRE turbulence, while $a=2$ means that the turbulence was artificially enhanced twofold for the fullwave computation.

In this scenario, which corresponds to the non-experimental electron temperature profile, it can be seen that with the growth of the turbulence amplitude, DR spectra shift to higher frequencies.
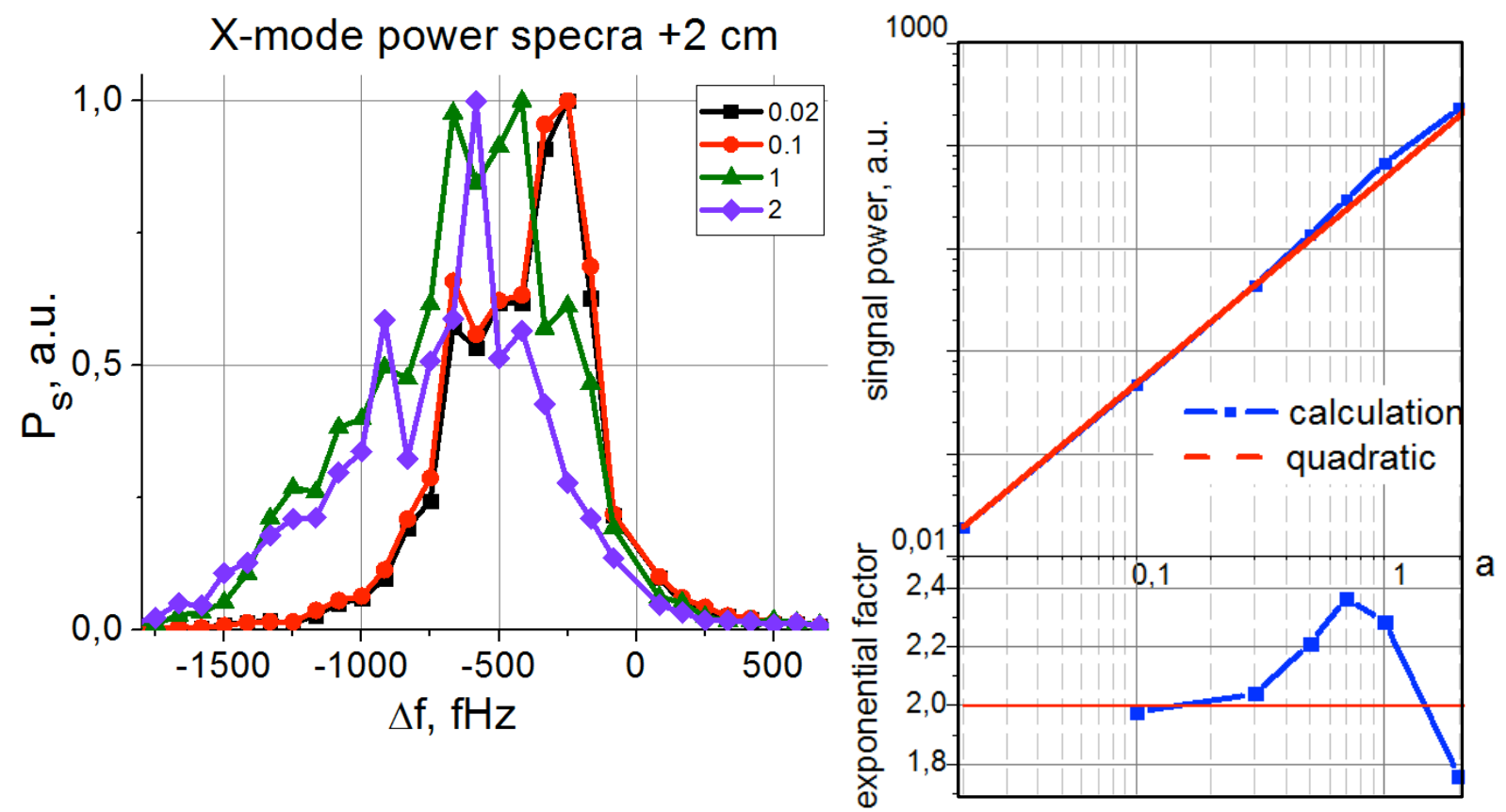

Fig. 1. DR signal power spectra for X-mode probing at Fig. 2. DR signal power dependence on the antenna vertical shift $+2 \mathrm{~cm}$. turbulence amplitude and exponential power factor.

The notion of this shift being caused by a nonlinear effect is further confirmed by the signal power growing at a rate different from quadratic for the higher $a$-values in the Fig. 2 . On the top half of the figure the scattered signal power is plotted. At about $a=0.5$ the growth becomes faster than linear, which corresponds to the nonlinear regime, described in [4, 5], while for $a$ values of 1 and higher the nonlinear saturation described by the analytical theory [6,7] and shown in the computations [5] can be observed. To make the nonlinearity more noticeable, an exponential factor defined for two values of $a$ as $(\lg (P 2)-\lg (P 1)) /(\lg (a 2)-\lg (a 1))$ where $P$ is the scattering signal power is plotted at the bottom half of Fig. 2. In the case of quadratic 
dependence corresponding to the linear scattering regime this factor has the value of 2 , for the nonlinear regime this value is higher than 2 , while in the saturated nonlinear regime the value becomes lower than 2 .

Nonlinear scattering, however, does not by itself explain the shift of the scattering spectrum to higher frequencies. The explanation we propose also includes nonlinear dispersion of turbulent fluctuations leading to a lower phase velocity at the higher fluctuation poloidal wavenumbers observed in the GK computations, shown as green solid curve in Fig. 3. That curve on the figure corresponds to the direct computation of the density fluctuations poloidal spectrum from ELMFIRE data with consecutive computation of the mean frequency of each wavenumber spectral component. Mean frequency for all curves is calculated as the first moment of the spectrum $f_{\text {mean }}=\int \Delta f \cdot p_{s}(\Delta f) d \Delta f / \int p_{s}(\Delta f) d \Delta f$, where $p_{s}(\Delta f)$ corresponds to the spectral power at specific frequency. For the solid curve it can be seen that for the higher poloidal wavenumber values this mean frequency saturates and stop increasing. This behavior is consistent with the theory expectations [22] providing the following dependence of the drift wave phase velocity in the plasma reference system: $V_{\mathrm{ph}}=V_{\mathrm{dr}}\left(1+k_{\perp}^{2} \rho_{\mathrm{s}}^{2}\right)^{-1}$. The earlier onset of such saturation in comparison with what other curves (corresponding to synthetic signals) display is explained by the fact that due to the turbulence amplitude getting smaller at higher $k_{\theta}$ the numerical noise plays a bigger role and adds a systematic error shifting the value of mean frequency closer to zero.

It should also be mentioned, that, strictly speaking, the points on the Fig.3 correspond to different radial positions of the turning point, which depend on the probing poloidal wavenumber. However, in the previous work [15] with the means of ray-tracing and the weighting function, the difference in radial position was shown to be up to $0.3 \mathrm{~cm}$ which is less than the weighting function localization and should not impact results significantly.

In the nonlinear regime characterized by multiple scattering, the backscattering signal is provided in part by fluctuations possessing a factor of 2 smaller poloidal wavenumbers than those satisfying the Bragg condition [5]. Therefore for the nonlinear turbulence dispersion law shown in Fig. 3 the multiple scattering off the lower-k fluctuations will result in a larger frequency shift than a single scattering induced by the fluctuation possessing a high poloidal wavenumber and satisfying the Bragg condition.

To confirm this idea, we considered the average DR signal spectrum frequency shift corresponding to the different antenna positions and consequently, different poloidal 
wavenumbers of the probing wave. If the proposed explanation is correct, in the linear regime we would see a saturation of the Doppler frequency shift with growing $k_{\theta}$ due to the turbulence dispersion law. However in the nonlinear regime of scattering we would obtain the dependence much closer to a linear one. This indeed turned out to be the case and the corresponding plot is shown in the Fig. 3, where the average frequency shift of scattered signal dependence are plotted along with the dispersion calculated directly from the turbulence. The quantities are plotted against the double poloidal wavenumber of the probing $\mathrm{X}$-mode in the turning point (corresponding to poloidal wavenumber of the turbulence) as well as against the antenna vertical shift $y$.
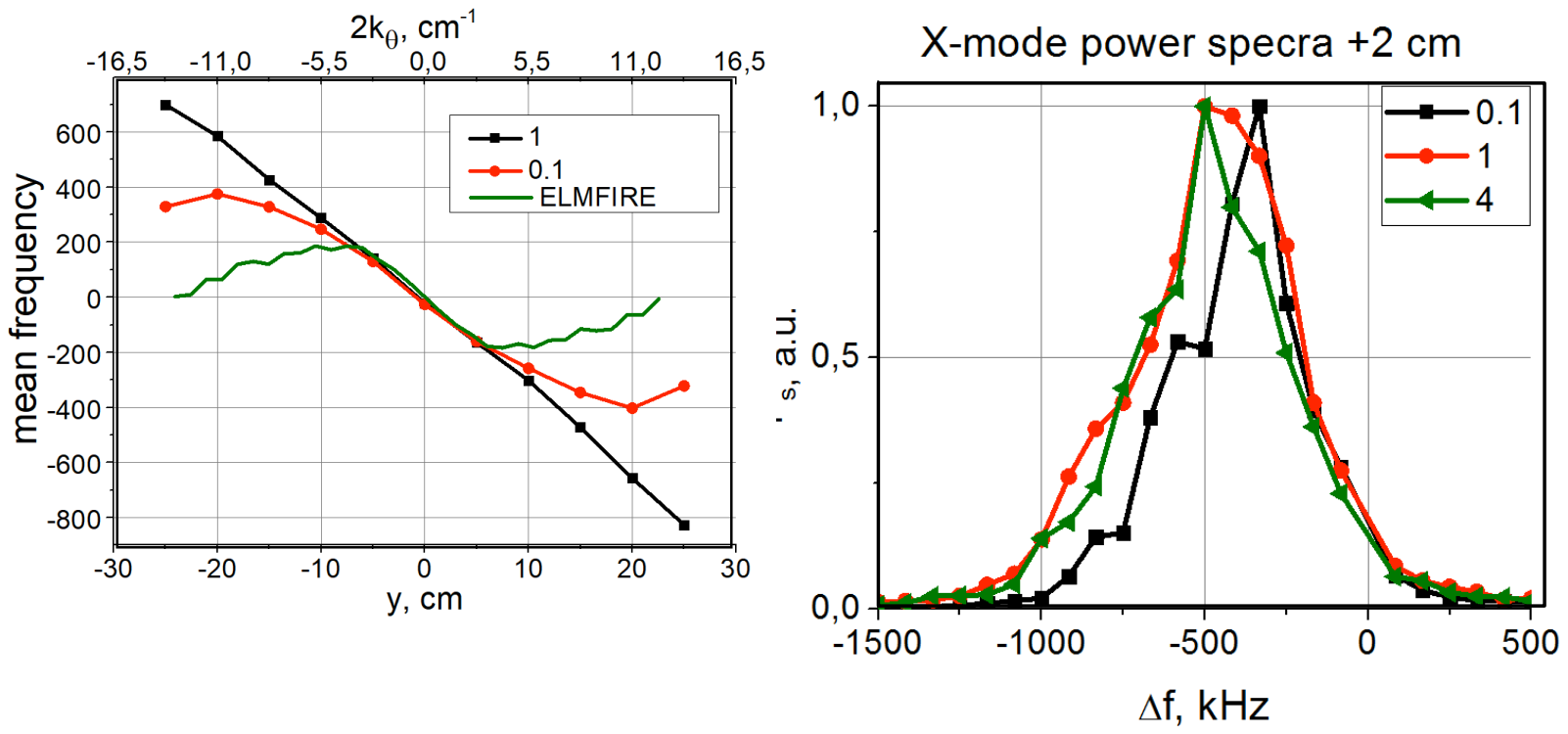

Fig. 3. Average frequency shift of scattering signal for X-mode against the double probing poloidal wavenumber on top and the vertical position of antenna $y$. Lines with markers correspond to

Fig. 4. Power spectra probing at antenna vertical shift $+2 \mathrm{~cm}$. Different lines correspond to different amplitudes of the turbulence. synthetic DR signal for different amplitudes of the turbulence. Solid line - the turbulence dispersion provided directly by ELMFIRE plotted against the turbulence poloidal wavenumber.

Next we present a modeling of DR signals utilizing the turbulence computed for the experimental profile of electron temperature [13]. This case is characterized by a slower decay of the poloidal wavenumber spectrum of the turbulence, which in turn means that nonlinear regime of scattering will now dominate signal at higher turbulence amplitudes, as predicted by the analytical theory [4],[5]. The spectra, obtained in this case for different turbulence levels and presented on Fig. 4, exhibit a substantially smaller frequency shift, while the DR signal power dependence on the turbulence level transits straight into the nonlinear saturation from the linear regime as it is seen in Fig. 5, which is a possibility 
predicted by the analytical theory and shown in the numerical computations [4], [5] for the case when turbulence spectrum is not steep enough.

The reason why the difference of spectra in this case is milder is probably the larger value of the linear Bragg backscattering signal, which dominates over the nonlinear one. Also, during the transition from a single to multiple scattering, the frequency shift does not change significantly (as seen in Fig. 6), likely due to the turbulence dispersion law, the saturation starts at higher $k_{\theta}$-values due to a smaller electron temperature. Unfortunately these values lie in the range where direct computation from ELMFIRE is impossible due to the strong numerical noise, as mentioned previously.

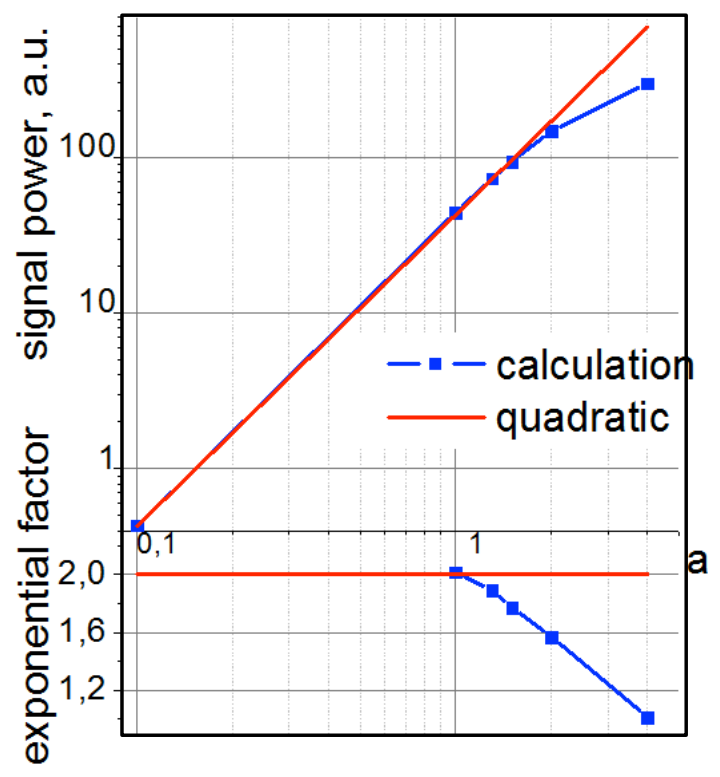

Fig. 5. DR signal power dependence on the turbulence amplitude and exponential power factor.

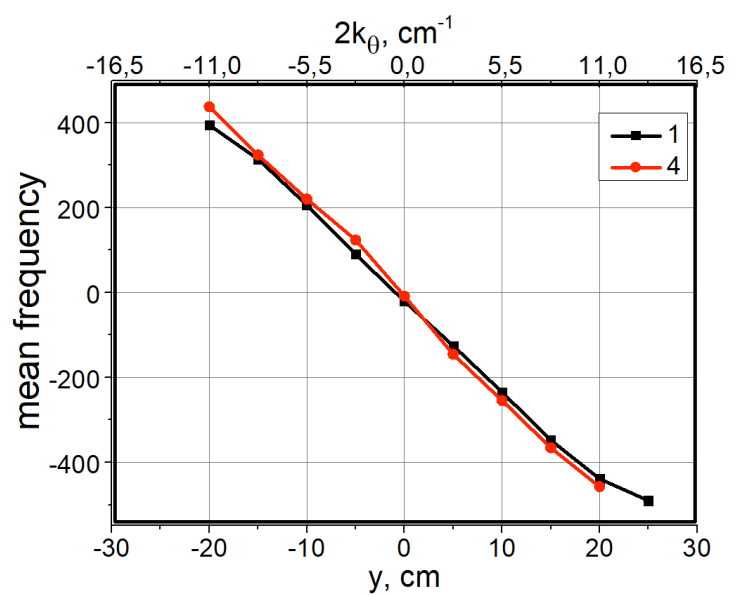

Fig. 6. Average frequency shift of scattered signal for X-mode. Different lines correspond to different amplitudes of the turbulence.

It should be mentioned that in this work we do not analyze the meaning of the obtained mean frequency shifts and their connection to Doppler shift or turbulence phase velocity. An extensive analysis of the mean frequency shift including rotation velocities, GAM influence [13] and impact of parallel velocity [15] is presented in previous works[16] and we refer the readers to these works for more information.

Overall it seems the main effect nonlinear regime has on the DR frequency spectrum is "linearization" of the Doppler frequency shift dependency on the probing wave poloidal wavenumber caused by the multiple scattering off the longer-scale turbulence dominating DR signal. The backscattering spectrum frequency shift in this regime is determined by the longscale turbulence dispersion law, as displayed at Fig. 3 and Fig. 6. This in turn means that the 
saturation of a linear dependence of the frequency shift could be considered as an indicator of the diagnostic operating in linear regime.

The effect of nonlinear scattering on the DR turbulence poloidal wavenumber spectrum measurements and on RCDR is well described in [6], and was reproduced in the computations $[12,14]$. In the case of DR signal power dependence on the antenna vertical shift $y$, which in the linear scattering regime is related to the turbulence poloidal wavenumber spectrum, the nonlinear effects lead to broadening of the dependence due to the nonlinear saturation of the spectral components corresponding to lower $k_{\theta}$ as it is shown on Fig. 7. In case of the RCDR $\mathrm{CCF}$, analytical theory predicts that in linear regime the correlation decay is slow due to the nonlocal small-angle scattering [2], [3] while in the nonlinear regime CCF does not directly correspond to the turbulence spatial correlation [6], [7] and was shown to be as steep or steeper than the turbulence correlation function [12]. A same effect was observed in this paper and is presented on the Fig 8. Both of these figures are plotted for the case of the experimental temperature values.
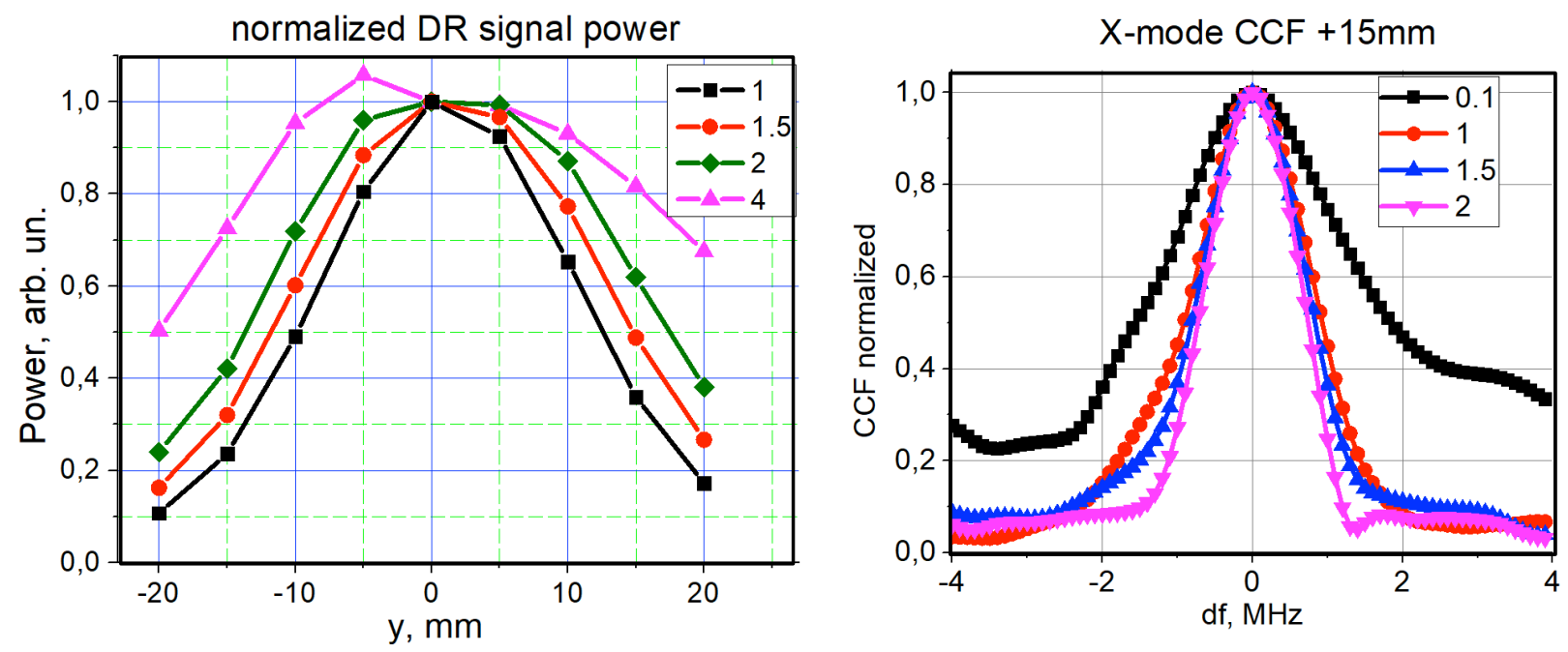

Fig. 7. DR power dependence on antenna vertical shift. Fig. 8. Normalized RCDR CCFs. Different lines

Different lines correspond to different amplitudes of the correspond to different amplitudes of the turbulence.

Both of the nonlinear effects observed are in agreement with the previous results [6],[7], [12], [14] and complicate the interpretation of experimental data. Moreover, obtained results indicate that RCDR diagnostic transits into the nonlinear regime at lower amplitudes of the turbulence compared to DR. Overall, is seems that to obtain reach? the correct interpretation of experimental data numerical modeling is necessary.

\section{O-mode DR modeling results and analysis}


For O-mode computation, Fig. 9 illustrates the nonlinear narrowing of the RCDR CCF with the turbulence amplitude growth, suggesting that the transition from the linear to the nonlinear regime is happening. This in turn means that The interpretation of the results coming from the original ELMFIRE data says that the RCDR operates in linear regime. This interpretation conclusion is further confirmed by the scattering signal power dependence on the turbulence amplitude shown in the Fig. 10.

As for the DR frequency spectrum, in the case of O-mode it still demonstrates the aforementioned shift to the higher frequencies in the nonlinear regime, but not a significant one, as can be seen at Fig.11. This is appropriate for "experimental"

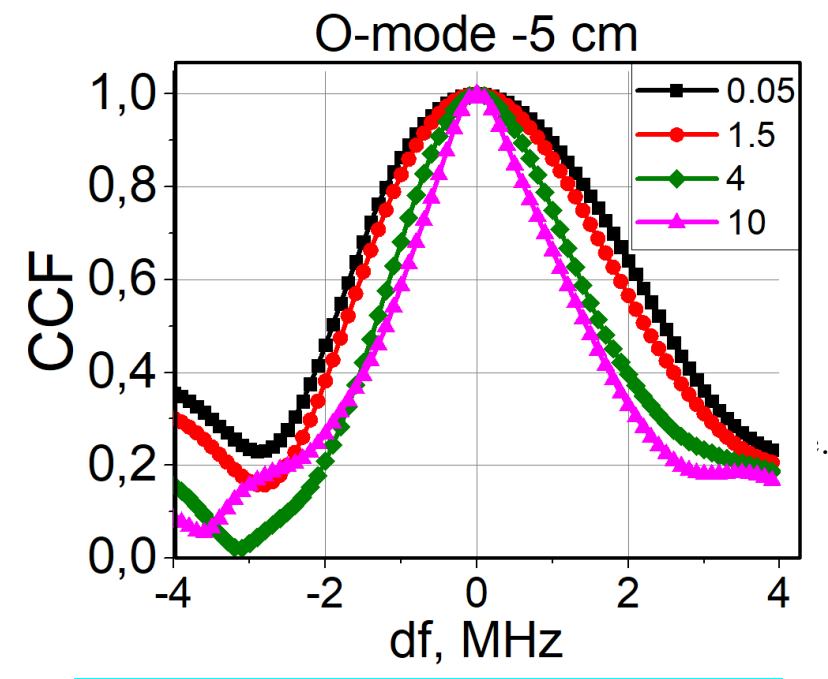

Fig. 9. CCF for $-5 \mathrm{~cm}$ antenna shift. Different lines correspond to different amplitudes of the turbulence.

ELMFIRE case, where the turbulence dispersion is closer to linear regime.

Nonlinear effects for O-mode start to play a role at much higher amplitudes compared to Xmode.

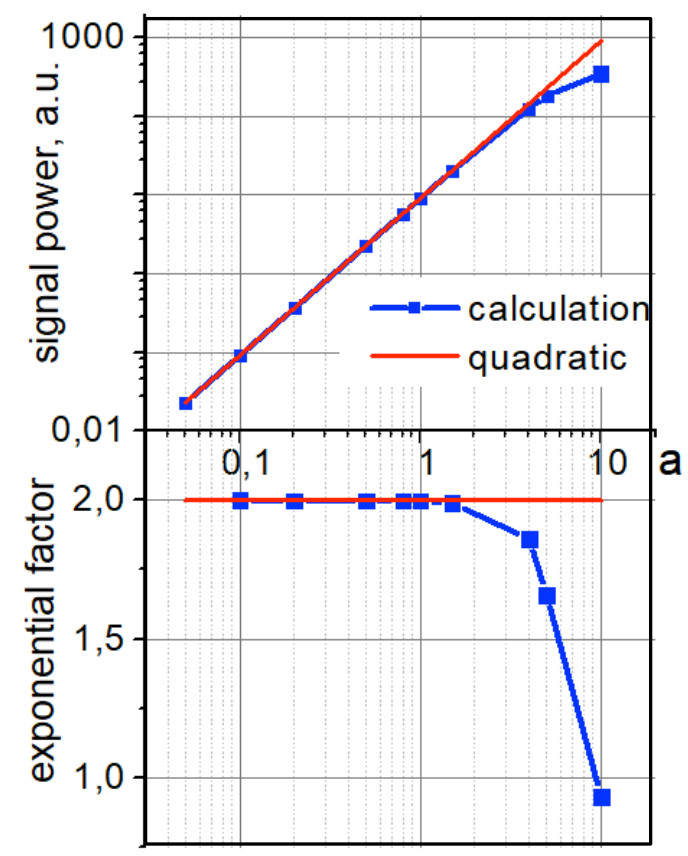

Fig. 10. DR signal power dependence on the turbulence amplitude.

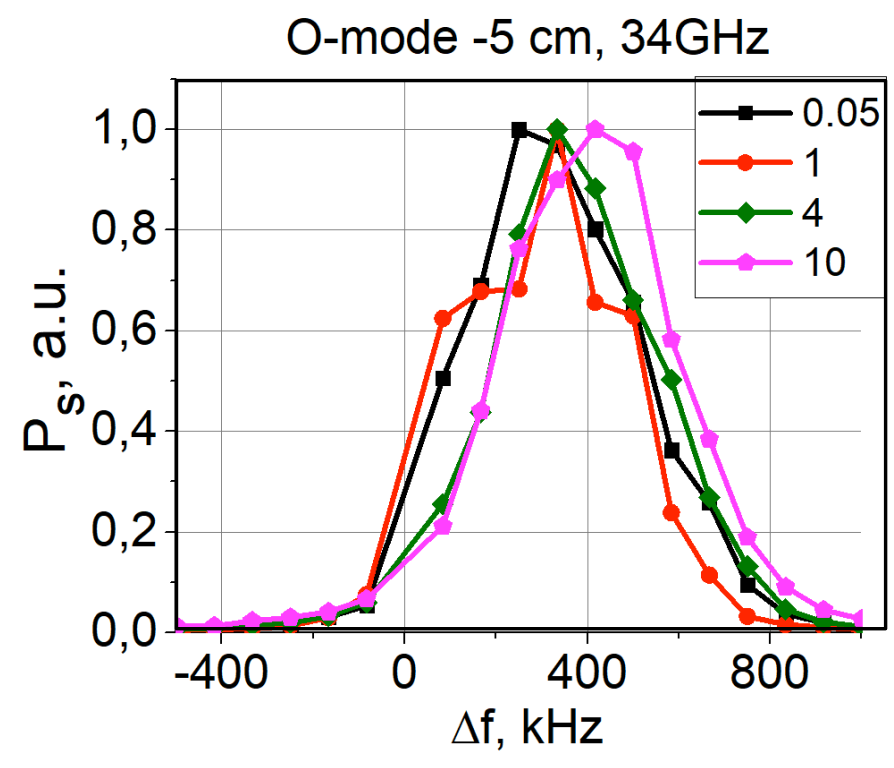

Fig. 11. DR signal power spectra for probing at $34 \mathrm{GHz}$ and different amplitudes of the turbulence.

Overall it seems that in the cases of O-mode for the experimental situation both DR and RCDR operate within the linear regime, which makes the linear numerical modeling relevant and means that $\mathrm{O}$-mode measurements are more suitable for a direct interpretation. 
While the results obtained have demonstrated a qualitative agreement with the analytical predictions, the subject of the future work would be to perform quantitative analysis on the nonlinear scattering regime offset. Analytical criteria provided by [4]-[7] would need to be adapted for the parameters domain of FT-2 experiments, possibly including modifications due to the cylindrical geometry effects and compared against the modeling results as well as other nonlinearity interpretations, such as those presented in [15] and [16].

\section{Conclusions}

Within this work the full-wave computations of synthetic DR and RCDR signals are performed using a realistic FT-2 tokamak turbulence obtained with the help of GK ELMFIRE code. Nonlinear effects are demonstrated and found to be in agreement with the theoretical expectations [4] [6][7]. An effect of "linearization" of the DR signal frequency shift dependence on the probing wavenumber is found in the nonlinear scattering regime. Nonlinear effects are shown to be weaker for O-mode probing in the studied cases, while a faster transition to the nonlinear regime is demonstrated for RCDR compared to DR in both probing scenarios.

\section{Acknowledgements}

Modeling of the O-mode DR signals was performed with the financial support of RFBR grant 18-32-00031. Modeling of the X-mode DR signals was performed with the financial support of the RSF grant 17-12-01110. Measurements at the FT-2 tokamak were supported by the state contract of the Ioffe Institute. This work has been partially carried out within the framework of the French Federation for Magnetic Fusion Studies (FR-FCM) and the EUROfusion Consortium and has received funding from the Euratom research and training programme 2014-2018 and 2019-2020 under grant agreement № 633053. The views and opinions expressed herein do not necessarily reflect those of the European Commission.

\section{References}

[1] J. Schirmer et al. Plasma Phys. Control. Fusion 49 1019. (2007)

[2] E. Z. Gusakov, A. Yu. Popov 38th EPS Conference on Plasma Physics P4.056 (2011)

[3] E. Z. Gusakov, M. A. Irzak and A. Yu. Popov Plasma Phys. Control. Fusion 56025009 (2014).

[4] J. R. Pinzon et al Plasma Phys. Control. Fusion 59035005 (2017)

[5] O. L. Krutkin et al Plasma Phys. Control. Fusion 61045010 (2019)

[6] E. Z. Gusakov, A.Yu. Popov Plasma Phys. and Control. Fusion 442327 (2002)

[7] E. Z. Gusakov and A. Yu. Popov Plasma Phys. Control. Fusion 461393 (2004)

[8] E. Z. Gusakov, A. V. Surkov and A. Yu. Popov Plasma Phys. Control. Fusion 47 959-974 (2005)

[9] E. Z. Gusakov and B. O. Yakovlev Plasma Phys. Control. Fusion 442525 (2002)

[10]E. Z. Gusakov and N. V. Kosolapova Plasma Phys. Control. Fusion 53045012 (2011)

[11]E. Z. Gusakov, M. A. Irzak, A. Yu. Popov et al. Physics of Plasmas 24, 022119 (2017)

[12]E. Blanco and T. Estrada Plasma Phys. Control. Fusion 55125006 (2013)

[13] O. Krutkin et al Nucl. Fusion 59096017 (2019)

[14]C. Lechte et al Plasma Phys. Control. Fusion 59, 07500 (2017)

[15]A. B. Altukhov et al Physics of Plasmas 25, 082305 (2018) 
[16]A. B. Altukhov et al Physics of Plasmas 25, 112503 (2018)

[17] A. B. Altukhov et al. Plasma Phys. and Control. Fusion 58105004 (2016)

[18] S. Leerink et al Phys. Rev. Lett., 109 165001, (2012)

[19]E. Z. Gusakov et al. 2013 Plasma Phys. Control. Fusion 55124034

[20] P. Niskala, PhD thesis, Aalto University, https://aaltodoc.aalto.fi/handle/123456789/34082 (2018)

[21] P. Niskala et al Plasma Phys. Control. Fusion 59, 044010 (2017)

[22] W. Horton 1999 Rev. Mod. Phys. 71735 (1999) 